\title{
The New Method of Lymphosorption for Patient's Treatment
}

\section{Shoikhedbrod $M^{*}$}

Electromagnetic Impulse Inc., Toronto, Ontario, Canada

\begin{abstract}
The use as the sorbents of activated carbon of different stamps does not give the possibility to extract from the lymph sufficiently the toxic metabolites, biologically active materials, unspecific cytoplasmic ferments, urea, creatinine, bilirubin, amylase, lipase, trypsin, ammonia and other. Furthermore, the existing processes of lymphosorption occupy much time, which is dangerous for the patient, whom necessary to live without the lymph, producing new and expecting purified lymph. The presence in lymph of the oncological patient the tumor cells, which become the sources of metastases in the lymph nodes, where the tumor cells intensely are reproduced because of the stagnancy of lymph and improved the conditions for the reproduction in these places. The necessity of the development of the new technologies of lymphosorption and methods of the destruction of tumor cells in the lymph of oncological patients is appeared. The paper presents the developed fundamentally new process of lymphosorption, which uses action on lymph of electric field and formed under this influence the negatively charged bubbles of hydrogen, and the specially designed everyday medical electroflotators that permit in short time to replace entire lymph of patient into the lymph of healthy person, freed from the cancerous cells, in the continuous regime, by recirculation on the locked outline.
\end{abstract}

Obtained experimental data permitted to establish the high effective influence of the developed process of lymphosorption on the treatment of patients of liver cancer and hepatitis of all forms. In connection with the fact that lymph is similar on its biochemical characteristics with the plasma and the blood, the developed process and electroflotators can be successfully used for removal from plasma and blood of toxic substances and cancerous cells. It is appropriate to use of the developed method of lymphosorption and the specially designed everyday medical electroflotators for treatment the patients.

Keywords: Lymphosorption; Lymphatic absorption; Hemosorption; Lymph, Blood; Plasma cleanse; Cancer

\section{Introduction}

The toxic lymph in the organism of patient can lead to the lethal outcome. Especially, this concerns the category of the patients of liver cancer and patients with the hepatitis of all forms, in which as a result of the defeat of the liver, the lymph contains repeated increase of bilirubin, urea, uric acid and alkaline phosphatase. In this case, the cleaning of lymph (lymphosorption) is critically important for these patients. The use of lymphosorption is vitally necessary for the detoxication of the cancer or hepatitis patients [1-7].

A decrease of the level of the laboratory parameters of endotoxicosis (bilirubin, transaminase, the molecules of average molecular weight, creatinine), and also the positive dynamics of the parameters of cellular immunity (content of lymphocytes) occurs in the process of lymphosorption. Lymphosorption is accompanied by an improvement of the parameters of central and peripheral blood flow. The use of lymphosorption helps to attain positive laboratory and clinical dynamics, to improve the results of treatment and to decrease lethality.

The basic method of cleaning lymph is the lymphatic drainage method of the purification of organism from the toxic metabolites, based on the removal of the lymph, which contains toxic substances, through the cannulated breast duct, and by the subsequent lymphosorption. The lymphosorption is achieved using the coal-sorbents of different stamps [1]. The degree of reduction of the toxic properties of lymph and contained metabolites after purification serves by the effectiveness evaluation criterion for absorption. Lymphosorption is conducted by several methods: dropping, in the statics and by recirculation on the locked outline.

Use as the sorbents of activated carbon of different stamps does not give the possibility to extract from the lymph of a sufficient measure toxic metabolites, biologically active materials, unspecific cytoplasmic ferments, urea, creatinine, bilirubin, amylase, lipase, trypsin, ammonia and other. Furthermore, process occupies much time, which is dangerous for the patient, whom necessary to live without the lymph, producing new and expecting purified lymph. It should note also about the presence in lymph among the oncological patients tumor cells, which become the sources of metastases in the lymph nodes, where the tumor cells intensely are reproduced because of the stagnancy of lymph and improved the conditions for these cells reproduction. In connection with the state above, the necessity of the development of the new technologies of lymphosorption and methods of the destruction of tumor cells in the lymph of oncological patients appeared.

The study of the action of electric field on the vitally important for the patient liquids, which are contained in his organism (lymph, plasma, blood), can lead to the creation of the completely new technologies, which can play revolutionary role in the treatment of patients.

\section{Materials}

The lymph, in the process of lymphatic drainage, was collected among the patients of liver cancer (15) in the Republic Clinical

*Corresponding author: Shoikhedbrod M, Electromagnetic Impulse Inc., Toronto, Ontario, Canada, Tel: 6509065; E-mail: mshoikh@primus.ca

Received November 14, 2017; Accepted November 29, 2017; Published December 02, 2017

Citation: Shoikhedbrod M (2017) The New Method of Lymphosorption for Patient's Treatment. J Blood Lymph 7: 190. doi: 10.4172/2165-7831.1000190

Copyright: (c) 2017 Shoikhedbrod M. This is an open-access article distributed under the terms of the Creative Commons Attribution License, which permits unrestricted use, distribution, and reproduction in any medium, provided the original author and source are credited. 
Oncological Dispensary (RCOD, Dushanbe, Tajikistan). The collected lymph contained $95 \%$ of water.

The bilirubin of the lymph (yellow microparticles) was increased and composed from 250 to $300 \mathrm{mcmol} / \mathrm{l}$. The urea of the lymph (white crystalline powder) was increased and composed from 69 to 79 $\mathrm{mcmol} / \mathrm{l}$. The uric acid of the lymph (colorless crystals) was increased and composed from 500 to $550 \mathrm{mcmol} / \mathrm{l}$. The alkaline phosphatase of the lymph (ferment microparticles) was increased and composed from 330 to $360 \mathrm{mcmol} / \mathrm{l}$. Therefore, the lymph with all important components inside presents the water compound with microparticles, whose concentration is repeatedly increased among cancer or hepatitis patients.

\section{Method and Developed Devices}

The lymph with all important components inside presents the water compound with microparticles and cancerous cells, whose concentration is repeatedly increased among cancer or hepatitis patients. Therefore, the primary task of lymphosorption is reduction of the concentration of these components and cancerous cells due to the separation and the removal from the lymph of the microparticles of these components and cancerous cells. The completely new technological process of lymphosorption, which uses action of DC (direct current) on the lymph, was developed $[2,3]$. Under the action of electric field on the lymph the negatively charged bubbles of hydrogen are formed on cathode. The size of formed negatively charged hydrogen bubbles can be controlled and can be as small, as this requires the technological process of lymphosorption.

The use of negatively charged with calculated dispersiveness of hydrogen bubbles permits to solve the primary task of the technological process of lymphosorption, connected to the removal of concentrations of microscopic particles and cancerous cells from lymph, the creation of strong complex of the hydrogen bubbles+particle of the solid component of lymph or cancerous cell in the process of the elementary act of floatation. After formation and detachment from the surface of cathode, the charged negatively bubbles of hydrogen, rising up in the lymph; meet on its way the particle of the solid component of lymph and cancerous cell by the dimensions of considerably larger than single bubble and bubbles induce the positive charge on the area of the particle of solid material or cancer cell.

As a result of the attraction of opposite charges, the bubbles stick to the particle or cancer cell, forming strong contact, and volume of formed complex of particle or cancerous cell+hydrogen bubbles strongly increases, and under the action of the considerably increased Archimedes force, the complexes of particle or cancerous cell+hydrogen bubbles with the increased speed floats upward. For conducting of the developed method of lymphosorption the special laboratory medical electroflotator was developed, whose common form is presented on Figure 1.

The special laboratory medical electroflotator presents the cylindrical container from the organic glass with inside diameter of 2.0 $\mathrm{cm}$, by height $11 \mathrm{~cm}$, whose bottom (with square $\mathrm{S}=3.8 \mathrm{~cm}^{2}$ ) is made from the stainless steel and serves as the cathode. The anode, made in the form of ring from the stainless steel, was fixed at a distance by $5 \mathrm{~mm}$ from the cathode. Electroflotator was supplied by the manual separator in the upper part of electroflotator for the selection of the concentrate of microsolid particles of the components of the lymph and cancerous cells, obtained during the process of the lymphosorption, in the receiver collector. The volume, which fills electroflotator by lymph, was about $40 \mathrm{~cm}^{3}$.

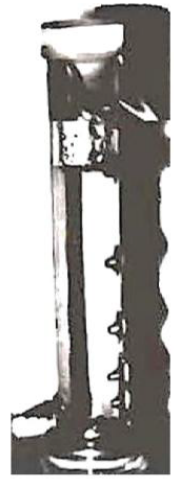

Figure 1: The common form of laboratory medical electroflotator

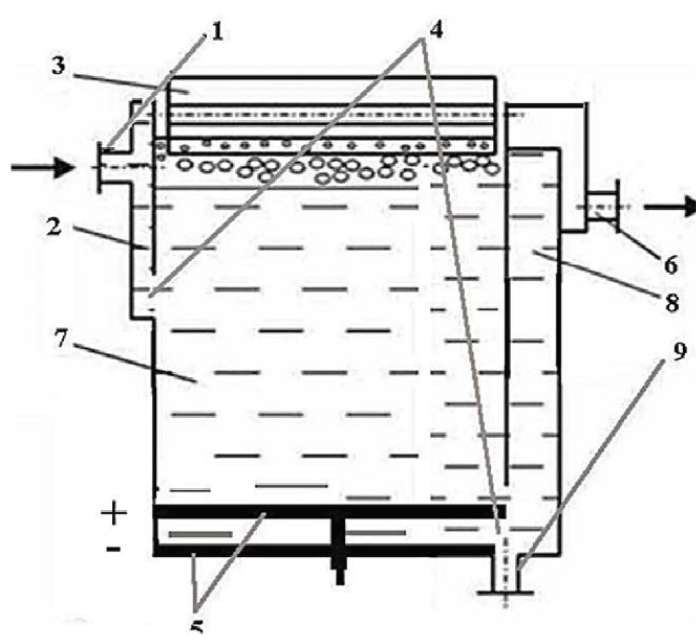

Figure 2: Special everyday medical electroflotator for the lymphosorption of continuous action.

Thus, the construction of electroflotator of this form is electroflotator of discrete action. The tests were conducted in the discrete, discontinuous regime. The lymph, in the process of lymphatic drainage, was selected from patient and was filled from top of the electroflotator. For conducting the tests the purified lymph was derived through the pin valve of electroflotator, located on the bottom of electroflotator. For the realization of lymphosorption for recirculation on the locked outline the special everyday medical electroflotator of continuous action was developed (Figure 2).

Structurally developed everyday medical electroflotator has floatation camera-7, fulfilled in the form of the rectangular capacity, whose angles are supplied by the special inserts, in consequence of which the internal part of the camera acquires the form of cylinder, and rear upper wall is supplied by the reflector. In the process of drainage, the selected among patient lymph, which contains all increased microcomponents and cancerous cells, using the special medical hose is entered to the floatation camera- 7 through the branch pipe with crane 1, pocket 2 and gap 4, for the realization of cleaning of the microcomponents from lymph and removal of cancerous cells. The purified lymph, after passing through the camera- 8 of additional cleaning, is moved away from electroflotator through the pocket and the drain branch pipe with crane- 6 and through the medical hose is returned to the patient for the recirculation on the closed cycle. 
The basic element of electroflotator is electrolytic base-5, fulfilled in the form of plug and of special mechanism, that permits simply to regulate by inter electrode gap size. The cathode was produced from the sterile stainless and is mounted on the bottom of camera. The anode was produced from the sterile corrosion-resistant metallic lattice uses the special construction which is located on the cathode that simply regulates the size of gap between anode and cathode. After completion of works for washing of electroflotator and output of the entire remained drain lymph the branch pipe with crane- 9 is provided. Crane, during the process of the work of electroflotator, is closed. The floated solid or liquid microcomponents of lymph, cancerous cells are collected in the foam layer in the upper part of the camera and are moved away by paddle device- 3 to the special capsule.

\section{Results}

The tests were conducted with the group of the patients of liver cancer on the base of Republic Clinical Oncological Dispensary (RCOD, Dushanbe, Tajikistan). The tests were conducted on the special constructed laboratory medical electroflotator of discrete action (Figure 1) in the discrete, discontinuous regime. The lymph, in the process of lymphatic drainage, was selected from patient and was filled from top of the electroflotator. The purified lymph was derived through the pin valve of electroflotator, located on the bottom of electroflotator, and was tested. The averaged results of tests for fifteen patients of liver cancer are presented in Table 1.

As follows from Table 1, after the lymphosorption by the developed method, all increased microcomponents of the lymph of the patients of liver cancer: bilirubin, urea, uric acid and phosphatase alkaline bounced back to the standard form of normal lymph of healthy person, suitable for the recirculation on the locked outline. The time of process composed only 15 seconds. The developed method of the lymphosorption is simple, rapid, economically advantageous (use a low currents and, therefore, low energy consumption) and harmless, since this method does not require the use of the expensive, harmful chemical reagents and method of centrifugation. For the purpose of the determination of the influence of the developed technological process of lymphosorption on the cancerous cells the experiments on the base of the company Israel Oceanographic and Limnological Research Ltd (Israel), where were used the several different strains of chlorella of the Red Sea (Eylat), were conducted [2]. The chlorella cells on their structure are identical to the cancerous cells.

The experiments were conducted on the special constructed laboratory medical electroflotator of discrete action (Figure 1) in the discrete, discontinuous regime. The microbiologists of the company Israel Oceanographic and Limnological Research Ltd noted, that the described method of the extraction of chlorella is simple, rapid, economically advantageous (use a low currents and, therefore, low energy consumption) and harmless, since this method does not require the use of the expensive, harmful chemical reagents and method of centrifugation. Nutrient medium, freed from the chlorella cells as a result of the process of concentration, could be used repeated. Thus, the results of experiments proved that the developed method of the

\begin{tabular}{|l|c|c|c|c|}
\hline Parameters & $\begin{array}{c}\text { Bilirubin, } \\
\text { mcmol/I }\end{array}$ & $\begin{array}{c}\text { Urea, } \\
\text { mcmol/I }\end{array}$ & $\begin{array}{c}\text { Uric acid, } \\
\text { mcmol/l }\end{array}$ & $\begin{array}{c}\text { Alkaline } \\
\text { phosphatase, unit/l }\end{array}$ \\
\hline Initial lymph & $250-300$ & $69-79$ & $500-550$ & $330-360$ \\
\hline Clean lymph & $5-10$ & $5-7$ & $250-300$ & $50-100$ \\
\hline Norm & $3-17$ & $2.8-7.2$ & $210-420$ & $30-120$ \\
\hline
\end{tabular}

Table 1: Results of lymphosorption by the developed method (time of the process -15 seconds). lymphosorption can be used for the extraction of cancerous cells from the lymph.

For the use of the developed lymphosorption in real medical practice for recirculation on the locked outline the special everyday medical electroflotator of continuous action was developed (Figure 2). The developed electroflotators are mobile and compact, which makes their use for patient comfortable in everyday life and permit by simple, rapid, economically advantageous and harmless method in short time to replace entire lymph of patient on the lymph of healthy person, freed from cancerous cells, in the continuous regime, by recirculation on the locked outline.

In process of tests with different strains of chlorella the microbiologists of the company Israel Oceanographic \& Limnological Research Ltd also noted that extracted chlorella cells remain living in the post process medium and intensely reproduced. An increase of the chlorella cells as the minimum 100 times is fixed. This result has the following explanation. As a result of the process of the concentration of chlorella the strong saturation of nutrient medium, which includes predominantly water, by hydrogen bubbles occurs, which leads to the creation so called "live water", which promotes to the intensive reproduction of chlorella cells. If the technological process is changed on the reverse process, i.e., the nutrient medium, with remaining chlorella cells, will be saturated by the positively charged oxygen bubbles, then possible to destroy the remaining chlorella cells.

In this case the "dead water" is formed, which leads to the destruction of chlorella cells. Thus, it can be developed a new technological method and constructed the corresponding electroflotator that permit to destroy remaining cancerous cells by subsequent saturation by the positively charged bubbles of oxygen of the purified after cleaning lymph in this electroflotator. In connection with this, the special everyday medical electroflotator (Figure 3 ) for the destruction of the cancerous cells, which is remained in the lymph after cleaning, by saturation by the positively charged bubbles of oxygen of the purified lymph was constructed.

Structurally the developed special everyday medical electroflotator has floatation camera-7, fulfilled in the form of the rectangular capacity, whose angles are supplied by the special inserts, in consequence of which the internal part of the camera acquires the form of cylinder, and rear upper wall is supplied by the reflector. The purified lymph, which contains the remained after lymphosorption cancerous cells, using the special medical hose, is entered to the floatation camera-7 through the branch pipe with crane 1, pocket 2 and gap 4, for the destruction of cancerous cells.

The purified lymph with the remained cancerous cells, after passing through the camera- 8 of additional cleaning, is moved away from electroflotator through the pocket and the drain branch pipe with crane- 6 and through the medical hose is returned to the patient for the recirculation on the closed cycle.

The basic element of electroflotator, in this case, was not only electrolytic base 5, fulfilled in the form of plug, and the special membrane-10, made from material of the type of fire hose and located in the inter electrode gap (Figure 3).

The membrane prevents the penetration through it of the formed as a result of the action of direct electric current (DC) on the lymph of the positively charged bubbles of oxygen and also penetration to the floatation camera of the formed of the negatively charged bubbles of hydrogen. As a result, the purified lymph is saturated by the positively 


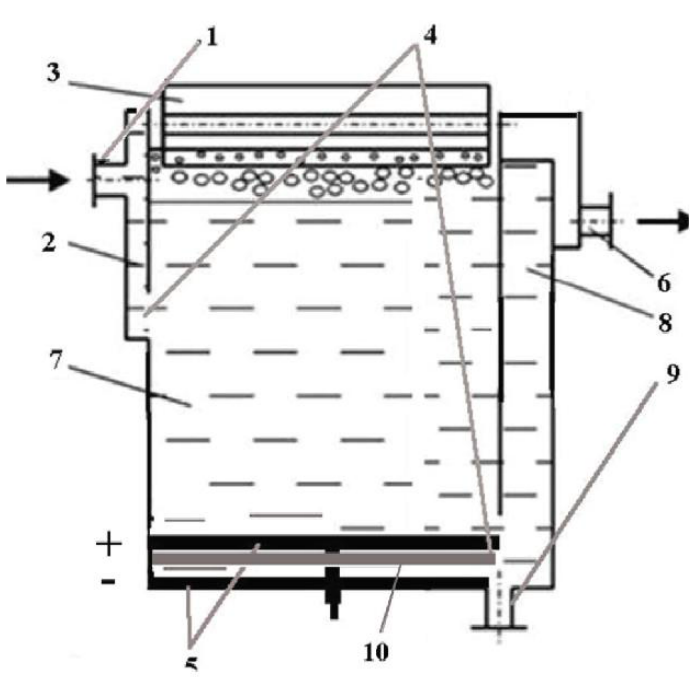

Figure 3: Special everyday medical electroflotator for the destruction of the cancerous cells of continuous action.

charged bubbles of oxygen, creating all conditions for the destruction of the remained cancerous cells. Entire purified from the remained cancerous cells lymph freely penetrates through the membrane and together with the hydrogen bubbles, after passing the camera 8 of additional cleaning, moves away from electroflotator through the pocket and the drain branch pipe with crane- 6 , and through the medical hose returns to the patient for the recirculation on the closed cycle.

After completion of works for washing of electroflotator and output of the entire remained drain lymph the branch pipe with crane- 9 is provided. Crane during the process of the work of electroflotator is closed. The floated remained solid or liquid microcomponents of lymph, killed remained cancerous cells are collected in the foam layer in the upper part of the floatation camera and are moved away by paddle device 3 to the special capsule. The developed everyday medical electroflotator is the electroflotator of continuous action and can work on the serial connection with other electroflotator for increasing the results of lymphosorption and destruction of cancerous cells in the lymph.

\section{Conclusion}

Thus, the developed process of lymphosorption and the specially designed everyday medical electroflotators permit in short time to replace entire lymph of patient on the lymph of healthy person, freed from cancerous cells, in the continuous regime, by recirculation on the locked outline. The developed electroflotators are mobile and compact, which makes their use for patient comfortable in practice. Obtained experimental data permitted to establish the high effective influence of the developed process of lymphosorption on the treatment of patients of liver cancer and hepatitis of all forms. In connection with the fact that lymph is similar on its biochemical characteristics to the plasma and the blood, the developed process and electroflotators can be successfully used for removal from plasma and blood of toxic substances and cancerous cells.

\section{References}

1. Shapko IP (2004) Lymphosorption through the shear of the placenta of human at the treatment of surgical pyoseptic diseases. Dissertations Candidates of Medical Sciences, Chelyabinsk.

2. Shoikhedbrod MP (2017) Behavior of water under the influence of vibration and electric field. Lambert Academic Publishing, Toronto.

3. Shoikhedbrod MP (2017) Computer modeling and the new technologies in oncology. Lambert Academic publishing, Toronto.

4. Margaris KN, Black RA (2012) Modeling the lymphatic system: challenges and opportunities. J R Soc Interface 9: 601-612.

5. Attili-Qadria S, Karra N, Nemirovski A, Schwob O, Talmon Y, et al. (2013) Oral delivery system prolongs blood circulation of docetaxel nanocapsules via lymphatic absorption. Proc Natl Acad Sci U S A 110: 17498-17503.

6. Ee LC, Zheng S, Yao L, Tso P (2000) Lymphatic absorption of fatty acids and cholesterol in the neonatal rat. Am J Physiol Gastrointest Liver Physiol 279: G325-G331.

7. Tsai T, Chen I (2010) Rebuttal to a Comment on Lymphatic Absorption of Quercetin and Rutin in the Rat and Their Pharmacokinetics in Systemic Plasma. J Agric Food Chem 58: 8468-8469. 